\title{
Oesophageal atresia and Down syndrome
}

\author{
Sebastiano Bianca ${ }^{\text {a-b }}$, Marco Bianca ${ }^{a}$ and Giuseppe Ettore ${ }^{a-c}$ \\ a Registro Siciliano Malformazioni Congenite, Catania, Italy \\ ${ }^{b}$ Dottorato di Ricerca in Malattie Genetiche dell'Età Evolutiva, Università di Catania, Catania, Italy \\ 'Divisione di Ostetricia e Ginecologia, Azienda Ospedaliera "Garibaldi”, Catania, Italy
}

\begin{abstract}
Infants with Down syndrome are known to have a high frequency of associated birth defects and some authors have suggested an association between Down syndrome and oesophageal atresia. We evaluated data from the Sicilian Registry of Congenital Malformations. Our finding of an incidence of $0.9 \%$ of oesophageal atresia in children with Down syndrome is more than 30 times higher than expected and more than reported in other studies where the association was present in $0.5 \%$ of cases. Our results confirm that the relationship between Down syndrome and oesophageal atresia is a non random association and the observation of this association in several populations with different genetic backgrounds allows us to conclude that a causal relationship may exist between Down syndrome and oesophageal atresia.
\end{abstract}

Keywords: Down syndrome, oesophageal atresia, upper alimentary tract anomalies, congenital malformations, birth defects, gene effects

Down syndrome is the most common chromosome anomaly recognised in man. Infants with Down syndrome are known to have a high frequency of associated birth defects. Oesophageal atresia is a congenital anomaly of upper alimentary tract, with a probable multifactorial aetiology, that occurs relatively frequently as an isolated malformation but it is often associated with other anomalies, sometimes as part of a specific syndrome.

Some authors have suggested an association between Down syndrome and oesophageal atresia. Prompted by evidence in the literature we reviewed our data to see whether there might be an association between these two conditions.

We evaluated data from the Sicilian Registry of Congenital Malformations (I.S.MA.C. Registry) database. From 1991 to 1998 in 243,916 live births, not including infants with Down syndrome, there were 90 cases of oesophageal atresia $(0.03 \%)$. During the same time period, 333 cases of Down syndrome were born alive $(0.13 \%)$, and 3 cases of oesophageal atresia were detected in this group $(0.9 \%)$.

Our finding of an incidence of $0.9 \%$ of oesophageal atresia in children with Down syndrome is more than 30 times higher than expected $(p<.0001)$ and more than reported in other studies where the association was present in $0.5 \%$ of cases (Torfs, Bateson \& Curry, 1992; Martinez-Frias, Urioste, Cereijo \& Rodriguez-Pinilla, 1992).

Our results confirm that the relationship between Down syndrome and oesophageal atresia is a non random association higher than expected by chance and according to the literature (Khoury, Cordero \& Rasmussen, 1988) there seems to be some specificity between the chromosome involved and congenital anomalies, as seems to be the case for oesophageal atresia. The relationship between chromosomal anomalies and congenital malformations may be then related to the time of embryo development in which the chromosomal abnormality produces its effect (MartinezFrias, et al, 1992).

A number of congenital malformations are clearly associated with Down syndrome and generally occur with a much higher incidence than in the general population (Källen, Mastroiacovo \& Robert, 1996). Understanding of the morphogenetic mechanisms involved in these anomalies is far from clear, in fact none of the anomalies associated with Down syndrome is specific to the trisomic state.

As suggested by Torfs and Christianson (1998) both the hypotheses of additive gene effects and of a general imbalance of the genome need to address the genetic mechanisms that explain both the increased frequency and the low frequency of specific defects among infants with Down syndrome.

The observation of this association in several populations with different genetic backgrounds allows us to conclude, as we have also done in connection with Down syndrome and anorectal malformations (Bianca \& Ettore, 2000), that a causal relationship may exist between Down syndrome and oesophageal atresia. 


\section{Correspondence}

Sebastiano Bianca • Registro Siciliano Malformazioni Congenite, Dipartimento di Pediatria, Via S. Sofia, 78 95123 - Catania, ITALY • E-mail:

sebastiano.bianca@tiscalinet.it

\section{References}

Bianca, S. \& Ettore, G. (2000). Anorectal malformations and Down's syndrome. Paediatic and Perinaalt Epidemiology, 14,372

Källen, B., Mastroiacovo, P., Robert, E. (1996) Major congenital malformations in Down syndrome. American Journal of Medical Genetics, 56, 160-166

Khoury, M.J., Cordero, J.F., Rasmussen, S. (1988). Ectopia cordis, midline defects and chromosomal abnormalities: An epidemiologic perspective. American Journal of Medical Genetics, 30, 811-817

Martinez-Frias, M.L., Urioste, M., Cereijo, A., Rodriguez-

Pinilla, E. (1992) Anorectal and Esophageal anomalies in Down syndrome. American Journal of Medical Genetics, $44,848-849$

Torfs, C.P., Bateson, T.F., Curry, C.J.R. (1992). Anorectal and Esophageal anomalies with Down syndrome. American Journal of Medical Genetics, 44, 847

Torfs, C.P. \& Christianson, R.E. (1998). Anomalies in Down syndrome individuals in a large population-based registry. American Journal of Medical Genetics, 77, 431-438 